\title{
The Modelica Thermal Model Generation Tool for Automated Creation of a Coupled Airflow, Radiation Model and Wall Model in Modelica
}

\author{
Arnav Pathak, Victor Norrefeldt, Abdellah Lemouedda, Gunnar Grün \\ Fraunhofer Institute for Building Physics, Dept. Indoor Climate, 83626 Valley, Germany \\ arnav.pathak@ibp.fraunhofer.de, victor.norrefeldt@ibp.fraunhofer.de, \\ abdellah.lemouedda@ibp.fraunhofer.de,gunnar.gruen@ibp.fraunhofer.de
}

\begin{abstract}
This paper presents the Modelica Thermal Model Generation Tool. The aim of this tool is to enable the user to set up a geometrically correct thermal model for complex geometries that allows predicting the impact of heated/heating devices and their location both in terms of airflow pattern and radiation distribution. Using a geometry file exported from CAD software, the tool distributes wall facets, air nodes and computes the long-wave radiant view factor matrix for obstructed and unobstructed surfaces. This information is exported as ready to use Modelica code. The zonal model VEPZO is used to model airflow within a domain (enclosed space). This model allows predicting airflow and air temperature distribution in space on a coarse mesh and thus computes faster than classical CFD computations. Walls are subdivided on the same grid as the zonal model is set upon. For each wall facet, the Modelica Thermal Model Generation Tool computes the view factors to the other facets in the domain.

Comparison of simulated results with test data and application of the Modelica Thermal Model Generation Tool for a room with radiant heating and for the cooling of an aircraft cockpit are presented in this paper.
\end{abstract}

Keywords: Thermal Model, VEPZO, Airflow Simulation, View Factor Calculation, Long-wave Radiant Heat Exchange, LowRad

\section{Introduction}

A number of codes for thermal simulation (e.g. Indoor Climate Library [1], Buildings Library [2]) have emerged in Modelica. These models are set up in a way to allow the user a comfortable parameterization with a moderate number of models. The resolution of these models is on room level. A typical model would consist of six rectangular enclosures, a perfectly mixed air volume and a radiation node estimating the view factors in a simplified way. Connecting a number of such basic elements and modelling an airflow network between rooms (e.g. the Multizone model of Buildings Library [3]) yields a building. To refine the airflow and temperature distribution within a room, zonal models have been implemented in Modelica ([1;4]). The manual setup of these models is easy when only rectangular spaces are considered. Simple nonrectangular spaces with a low number of inclined surfaces can still be set up manually, too. However, the manual setup of a thermal model for more complex geometries becomes increasingly time consuming and error prone with increasing "nonrectangularity" of the geometry as surfaces and zones require a one-by-one parameterization of area, volume and location. Furthermore, the simplified radiation approach distributing radiation proportionally on surfaces (e.g. described in [5]) is only validated for building applications where typically temperature differences between surfaces are relatively small. In other configurations, the simplified approach may result in high errors of surface temperatures due to wrong estimation of view factors, which can further cause error in energy balance and estimation of comfort level.

One of the purposes of creating the Modelica Thermal Model Generation Tool is to allow estimating the impact of the location of a very hot device or the development of local radiant or convective heating strategies.

To achieve this goal, the Modelica Thermal Model Generation Tool combines two Modelica codes presented at the Modelica Conference 2012. The VEPZO model [6] computes the airflow and temperature distribution in space. Up to now, it has only been used on rectangular geometries. For nonrectangular spaces, the geometry was manually approximated by rectangular elements. The Modelica Long-wave Radiation View Factor Model [7] allows 
for view factor computation based on actual geometries and creates a more detailed radiation node model. The long-wave radiation view factor model presented in Modelica Conference 2012, was not considering the obstruction check for obstructed views.

\section{Method}

This section outlines the steps of the Modelica Thermal Model Generation Tool and used models.

\subsection{Modelica Thermal Model Generation Tool}

The Modelica Thermal Model Generation Tool (MThMGT) is a pre-processing tool developed in $\mathrm{C}++$ language. It translates a CAD model exported into the .stl format to Modelica code that can be simulated in the Modelica simulation environment. The .stl format describes the geometry by a series of triangles and their normals. When opening the MThMGT GUI (Figure 1), the user selects the input .stl-file and determines a grid for the subdivision of the geometry into zones. The user can add heat sources and ventilation openings in the GUI.

Based on the user-defined grid, the MThMGT distributes nodes and checks for each node whether it is within or outside the geometry. Furthermore, it checks whether two adjacent nodes are separated by a wall indicating that they belong to two different domains (i.e. room 1 and room2). The nodes within one domain form the edges of the zones. The "classical" rectangular zone is thus determined by eight nodes. Zones adjacent to the geometry are defined by less than eight nodes as some nodes are outside the domain. For these zones, dedicated rules are applied approximating zone geometry and creating wall facets that approximate the actual geometry. Each zone is given a unique name to be identified for its connections to walls, heat sources, convection and radiation models and ventilation openings. To enable the user to assess the created thermal model, the geometry of created zones and wall facets is saved as .stl file in dedicated repositories. Figure 2 shows an example for the distribution of zones in geometry.

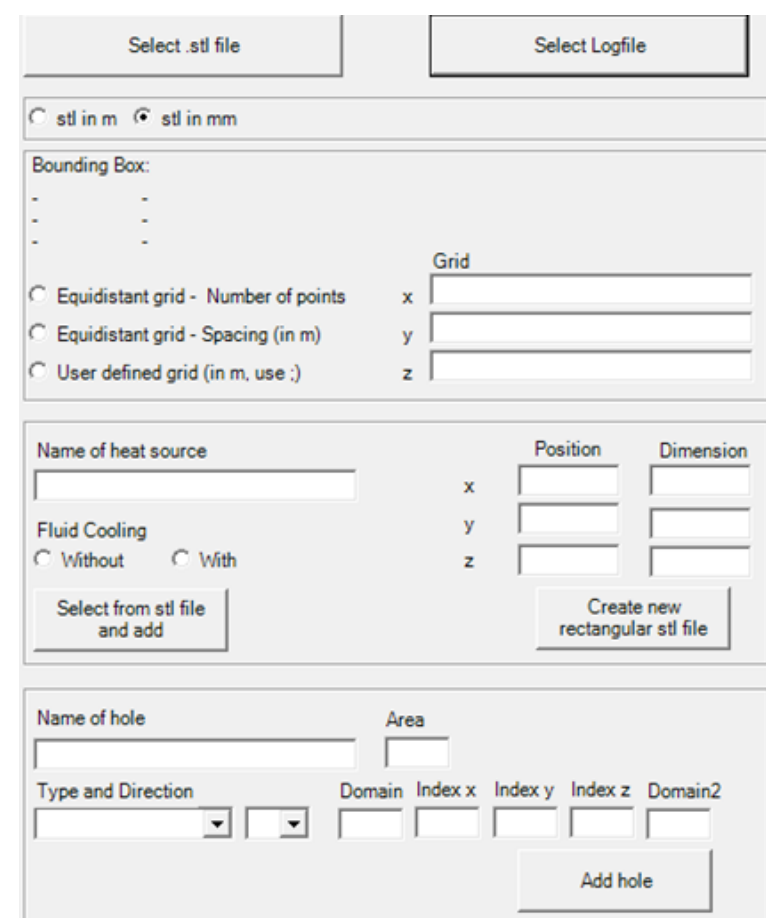

Figure 1: GUI of the MThMGT - Zonal Part

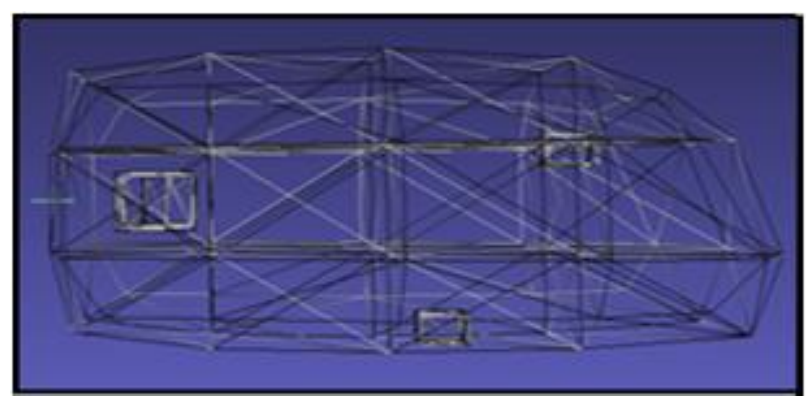

Figure 2: Example for created zonal grid

View factor computation settings are entered in the second part of the GUI of MThMGT (Figure 3). First, the user needs to state whether view factors shall be computed and what kind the geometry is of. Unobstucted view means all the surfaces in one domain have complete view of each other if they are not in the same plane. If there is any obstruction in the view of one surface to another surface, the user must select computation method for obstructed views. Similarly, if the surface is self obstructed, for example a surface is shaped as an alphabate ' $S$ ', the user must select the computation method for selfobstructed views. The aim of these selection possibilities is to obtain a quicker computation by avoiding unnecessary obstruction checks. 


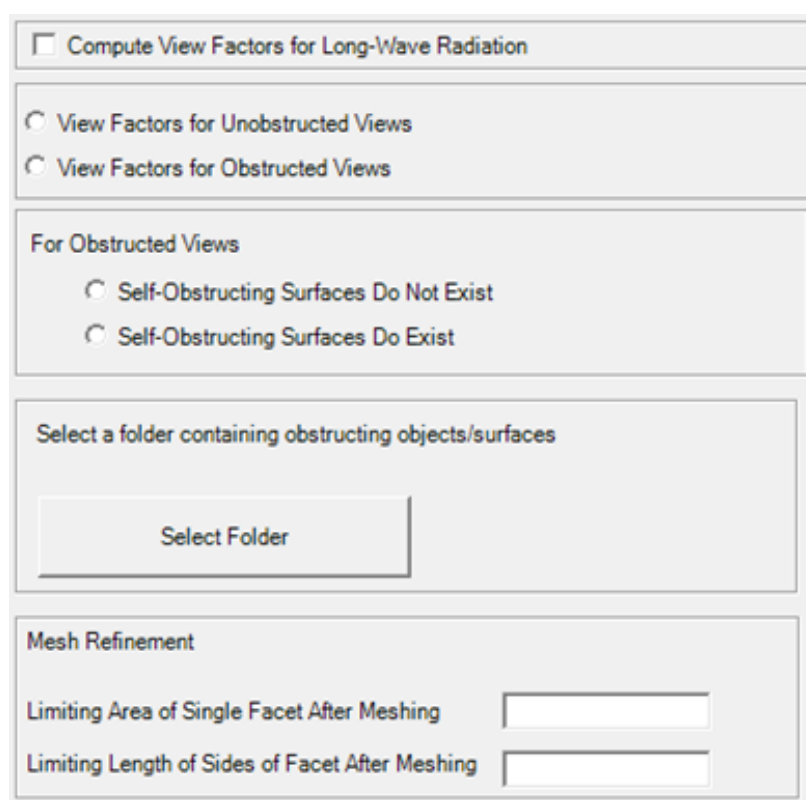

Figure 3: GUI of the MThMGT - Radiation Part

Accuracy of view factor computation is directly proportional to the mesh quality of a surface. Therefore, mesh refinement is required. For this, the user can define a limiting area and a limiting length of facet edges for refined triangles. This ensures that all facets are refined below this threshold. Figure 4 shows a refined mesh of the geometry shown in Figure 2.

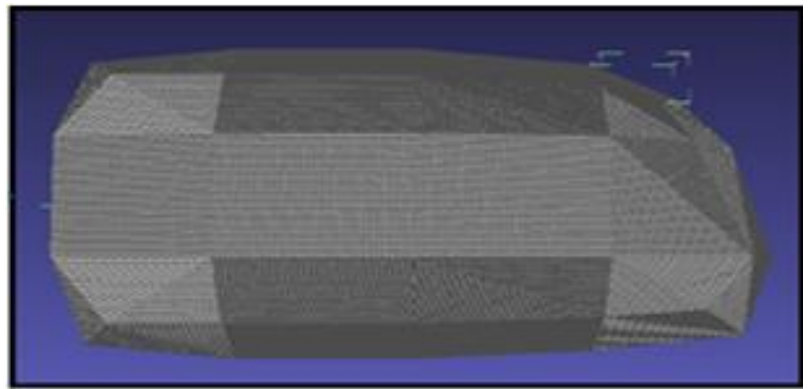

Figure 4: Refined meshing of created geometry for view factor computation.

After computation of zonal grid and view factors, the MThMGT does all the appropriate connections between the zonal model, the radiation model, the convection models and the wall models. It includes heat sources and ventilation openings to the model and connects them with the appropriate zone and radiation node. The MThMGT writes all the model descriptions and connections into a Modelica file (.mo file) which can be further used in a Modelica environment for thermal simulation.

\subsection{VEPZO zonal airflow model}

The two main components of the VEPZO model are a zone model and a flow model (Figure 5). The zone (cube) and the flow (grey rectangle) models are connected by ports to form a room. These ports allow the exchange of relevant information between the flow and the zone model. The flow models have two ports to connect adjacent zones. Each zone has six ports, one for each boundary. A boolean parameter is assigned to each port to make the distinction whether the port is connected to a flow model or whether there is no flow because the zone is adjacent to a room boundary surface. Furthermore, each zone has a heat port (red square) allowing heat exchanges with models of other components like heat sources or walls.

With the MThMGT, these connection parameters are automatically set and ports are automatically connected to the respective models.

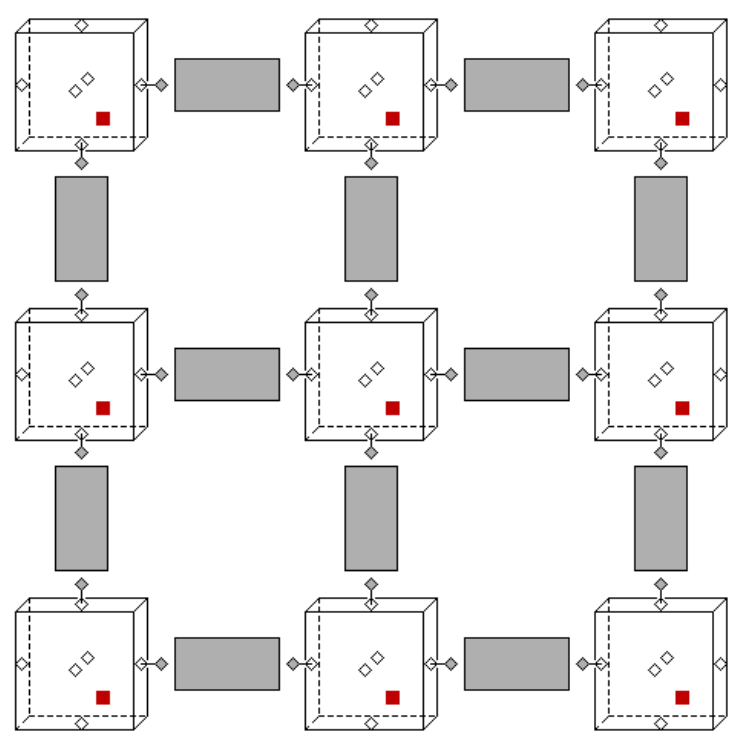

Figure 5: Zonal model in $x-z$ direction ( $y$ not shown); cubes: zones; grey rectangles: flows; rhombs: airflow ports; red solid squares: heat ports.

The main task of the zone model is to compute mass and energy balances and air properties (density, enthalpy, pressure, temperature, etc) using air models of Modelica.Media.

$\frac{\partial \rho_{i}}{\partial t}=\frac{\sum_{j} \dot{m}_{i, j}+\sum_{\text {sources }} \dot{m}_{\text {source }, i}}{V_{i}}$ 


$$
\begin{aligned}
& \mathrm{V}_{\mathrm{i}} \cdot \rho_{\mathrm{i}} \cdot \frac{\partial \mathrm{h}_{\mathrm{i}}}{\partial \mathrm{t}}=\sum_{\mathrm{j}} \dot{\mathrm{m}}_{\mathrm{i}, \mathrm{j}} \cdot \mathrm{h}_{\mathrm{i} / \mathrm{j}}+ \\
& \sum_{\text {sources }} \dot{\mathrm{m}}_{\text {source }} \cdot \mathrm{h}_{\mathrm{i} / \text { source }}+\sum_{\text {heatflows }} \dot{\mathrm{Q}}
\end{aligned}
$$

Where pressure is constant, $\mathrm{V}$ is the volume, $\rho$ is the density, $\mathrm{h}$ is the enthalpy, $\mathrm{m}$ are entering and leaving airflows, and $\mathrm{Q}$ are heat flows in the zone.

The main task of the flow model is to compute the airflow rate between two adjacent zones. For this, forces resulting from pressure, momentum and height difference and losses are summed yielding the acceleration of the airflow in the flow paths. A detailed set of equations for the flow path can be found in [6].

\subsection{Long-wave Radiant Heat Exchange Model: Low Rad}

The radiant heat exchange of a surface depends on the temperature $T$, the reflectivity $\rho$, the emissivity/absorptivity $\varepsilon$ and for transparent surfaces the transmissivity $\tau$.

For an opaque surface $\mathrm{i}$ the incoming $\left(\mathrm{Q}_{\mathrm{i}, \text { in }}\right)$ and leaving $\left(\mathrm{Q}_{\mathrm{i}, \text { out }}\right)$ radiant heat flows are computed by:

$$
\begin{aligned}
& Q_{i, \text { out }}=A_{i} \cdot \varepsilon_{i} \cdot \sigma \cdot T_{i}^{4}+\rho_{i} \cdot Q_{i, \text { in }} \\
& \mathrm{Q}_{\mathrm{i}, \text { in }}=\sum_{\mathrm{j}} \mathrm{F}_{\mathrm{i}-\mathrm{j}} \cdot \mathrm{Q}_{\mathrm{j}, \text { out }}
\end{aligned}
$$

with $\sigma$ : Boltzmann number $\left(5.67 \cdot 10^{-8} \mathrm{~W} / \mathrm{m}^{2} \mathrm{~K}^{4}\right)$, $F_{i-j}$ : view factor of surface $j$ to $i$.

Determination of the long-wave radiant heat exchange between surfaces requires the view factor matrix $F_{i-j}$. There are several analytical solutions available to calculate view factors for simple and known configurations. Many building simulation programs estimate the view factors in a simplified way, especially when complex geometries are involved. The simplified approach may result in high errors of surface temperatures, which can further cause error in energy balance. Hence it is important to determine accurate view factors. The purpose of creating this model is to calculate view factors between complex geometries for unobstructed, obstructed and self-obstructed views.

\subsubsection{View factor matrix integral}

To assess the radiative exchange between nonobstructed surfaces, the corresponding view factors between the surfaces need to be computed. View factors between two surfaces are dependent on the geometry of the surfaces and their orientation. The view factor can be interpreted as the fraction of diffusive radiant heat exchange between surface $\mathrm{i}$ and surface $\mathrm{j}$. The view factor between two surfaces is obtained from the integral over the view factors between the infinitesimal surface elements $\mathrm{dA}_{\mathrm{i}}$ and $\mathrm{dA}_{\mathrm{j}}$ (equation (5) and Figure 6):

$$
\mathrm{F}_{\mathrm{ij}}=\frac{1}{\mathrm{~A}_{\mathrm{i}}} \int_{A \mathrm{i}} \int_{\mathrm{Aj}} \frac{\cos \theta_{\mathrm{i}} \cdot \cos \theta_{\mathrm{j}}}{\pi \cdot \mathrm{r}^{2}} \mathrm{dA}_{\mathrm{i}} \mathrm{dA}
$$

where $r$ is the distance between the centres.

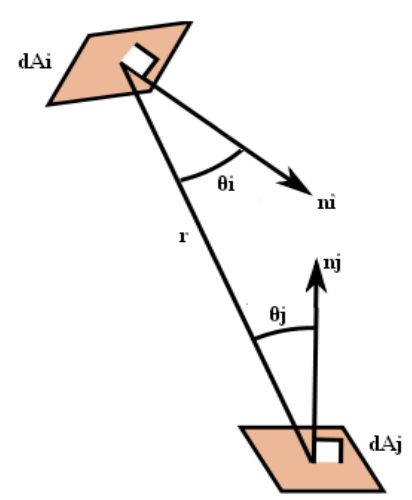

Figure 6: View factor between two surfaces i \& j

It is sufficient to compute the upper 1 triangle of the view factor matrix. The lower triangle can be constructed by the following relationship:

$$
A_{i} \cdot F_{i j}=A_{j} \cdot F_{j i}
$$

\subsubsection{View factor matrix computation}

The input for the computation of the view factor between two surfaces $i$ and $j$ is their triangulation in the .stl format. The MThMGT reads vertices and the normal vector of each triangular facet. The model calculates the centre and area of each triangle, the distance between each triangle and all the other triangles and similarly directional cosines for each triangle pair. For this, the discretization of equation (5) is used:

$$
F_{i j}=\frac{1}{A_{i}} \sum \sum \frac{\cos \theta_{i} \cdot \cos \theta_{j}}{\pi \cdot r^{2}} \cdot d A_{i} d A_{j}
$$

Required angles are determined by using following equations: 


$$
\begin{aligned}
& \cos \theta_{i}=\frac{l_{i}\left(x_{j}-x_{i}\right)+m_{i}\left(y_{j}-y_{i}\right)+n_{i}\left(z_{j}-z_{i}\right)}{r} \\
& \cos \theta_{j}=\frac{1_{j}\left(x_{i}-x_{j}\right)+m_{j}\left(y_{i}-y_{j}\right)+n_{j}\left(z_{i}-z_{j}\right)}{r}
\end{aligned}
$$

with $1, \mathrm{~m}, \mathrm{n}$ : directional components of the normals of $\mathrm{dA}_{\mathrm{i}}, \mathrm{dA}_{\mathrm{j}}$

$\mathrm{x}, \mathrm{y}, \mathrm{z}$ : coordinates of the centers of the surface elements.

Summing this discretized integral over all triangles forming the two surfaces yields the view factor between these two surfaces. Once the view factor $F_{i j}$ from surface $i$ to surface $j$ is known, the reciprocal view factor from surface $j$ to $i F_{j i}$ can be calculated from equation (6).

\subsubsection{Obstruction check}

To check whether two surface elements are obstructed by a third element, the plane of eventual obstruction needs to be set up.

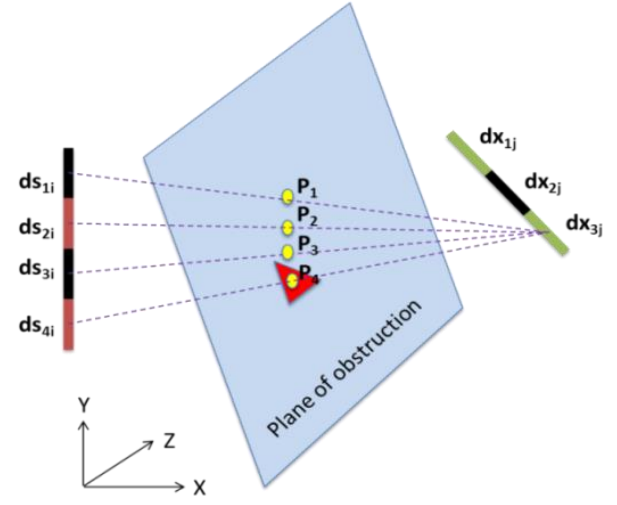

Figure 7: Obstructed view factor between surface $i$ and surface $j$

The equation of plane is given by

$$
\begin{aligned}
& A \cdot x+B \cdot y+C \cdot z+D=0 \\
& D=-\left(A \cdot x_{o b}+B \cdot y_{o b}+C \cdot z_{o b}\right)
\end{aligned}
$$

Where $\mathrm{x}_{\mathrm{ob}}, \mathrm{y}_{\mathrm{ob}}$ and $\mathrm{z}_{\mathrm{ob}}$ are the vertices of the obstructing triangular facet (in this case only one facet is obstructing the view). The equation of the line connecting centers of facets on surface-i (i.e. centers of $\mathrm{ds}_{1 \mathrm{i}}, \mathrm{ds}_{2 \mathrm{i}}, \mathrm{ds}_{3 \mathrm{i}}, \mathrm{ds}_{4 \mathrm{i}}$ ) and surface-j (i.e. centers of $\mathrm{dx}_{1 \mathrm{j}}, \mathrm{dx}_{2 \mathrm{j}}, \mathrm{dx}_{3 \mathrm{j}}$ ) checks the relative position between the line and the obstructing plane. There are three possibilities:

- The line can be parallel to the obstructing plane
- The line can belong to the obstructing plane

- The line can intersect the obstructing plane.

For this, the relative position of the obstructing surface to the line is checked:

$$
\begin{aligned}
& \mathrm{m}=\frac{\mathrm{A} \cdot \mathrm{x}+\mathrm{B} \cdot \mathrm{y}}{\mathrm{A} \cdot \mathrm{a}+\mathrm{B}} \\
& \left(\begin{array}{l}
\mathrm{a} \\
\mathrm{b} \\
\mathrm{c}
\end{array}\right)=\left(\begin{array}{l}
\mathrm{x}_{j}-\mathrm{x}_{\mathrm{i}} \\
y_{j}-y_{i} \\
z_{j}-z_{i}
\end{array}\right)
\end{aligned}
$$

If the plane of obstruction is between surfaces $i$ and $\mathrm{j}$, the value of $\mathrm{m}$ is greater than 0 and less than 1 .

If the denominator of equation (12) is not zero, the point $p$ of the intersection between the line and the plane can be found using:

$$
\left(\begin{array}{l}
x_{p} \\
y_{p} \\
z_{p}
\end{array}\right)=\left(\begin{array}{l}
x_{i}+a \cdot m \\
y_{i}+b \cdot m \\
z_{i}+c \cdot m
\end{array}\right)
$$

Once the point $p$ is known, the next step is to find out using barycentric technique if the point $p$ is inside the obstructing triangle or not. If the point $p$ is inside the triangular facet the view factor is assumed to be zero.

\subsubsection{Radiation node model}

The radiation node model calculates long-wave radiant heat exchange between $\mathrm{n}$ surfaces. It contains $\mathrm{n}$ thermal ports, surface properties and the view factor matrix. Each port connects to the corresponding surface model. The incoming and outgoing radiations for each surface are computed using the view factor matrix, temperature at each thermal port and surface properties as per equations (3) and (4).

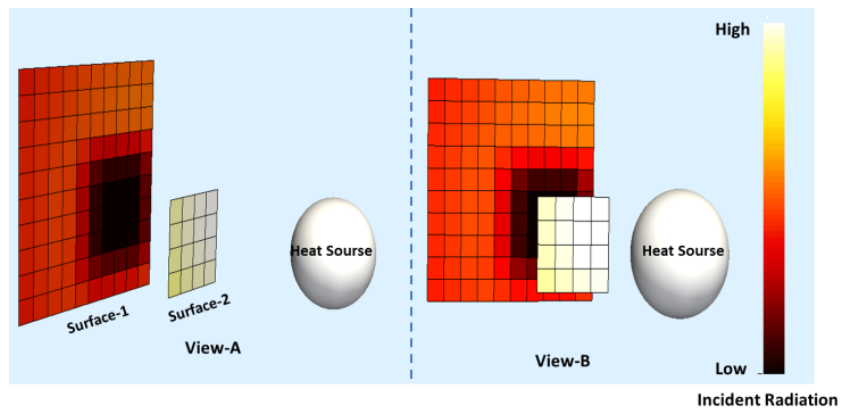

Figure 8: Incident radiation on two surfaces

Application example of radiation model with obstructed view can be seen in Figure 8. There are two surfaces in front of radiant heat emitter (Globe). 
Some part of surface- 1 is obstructed by surface- 2 . Different colors represent difference in intensity of incident radiation. Due to the lower distance from the globe to surface-2, it has a higher incident radiation than surface- 1 . The view factor of the globe to the obstructed part of surface-1 is zero. Hence there is no direct radiant heating of the obstructed part.

\subsection{Walls and convection models}

Convection models are used from the Modelica Standard Library. Wall models are based on a suite of thermal capacitances and thermal resistances from the Modelica Standard Library. The parameterization of these models yields the different materials that can be used.

The MThMGT exports the wall type for each domain with a "none" wall. This wall has no capacitance and no thermal resistance. In the exported code, the model type can be changed to the wall actually used in the geometry.

\section{Coupled Model Validation}

The coupled airflow, radiation and wall model generated with the MThMGT has been validated on a test bench.

\subsection{Test Setup}

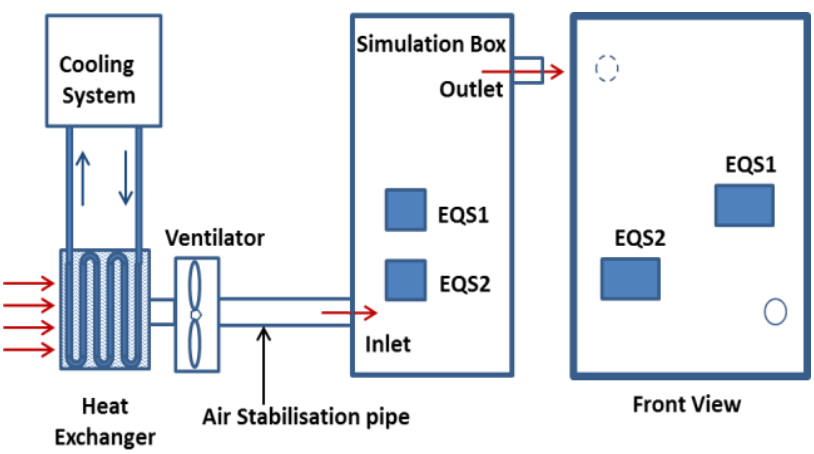

Figure 9: Experimental Setup: Box with air conditioning system

The experimental setup is composed of a box of size $2 \mathrm{~m} \times 1.5 \mathrm{~m} \times 0.9 \mathrm{~m}$ connected to an air supply system and insulated with $10 \mathrm{~cm}$ thick Armaflex foam. The box contains two heat sources in a form of equipment simulators (EQS) (Figure 9). These are installed in a test zone in a manner to have air circulation around them. By hanging them with a thin non-metallic rod their support can be considered to be thermally insulated. Heating foils release a uniform heat flow on all surfaces of the EQS.

Conditioned air is supplied to the simulation box with a pipe of $104 \mathrm{~mm}$ diameter. In order to get a well developped flow at the inlet a minimum pipe length of $\mathrm{L}=15 \mathrm{xD}_{\text {hydraulic }}$ is used. This well developped flow is also required for a high inlet velocity measurement accuray.

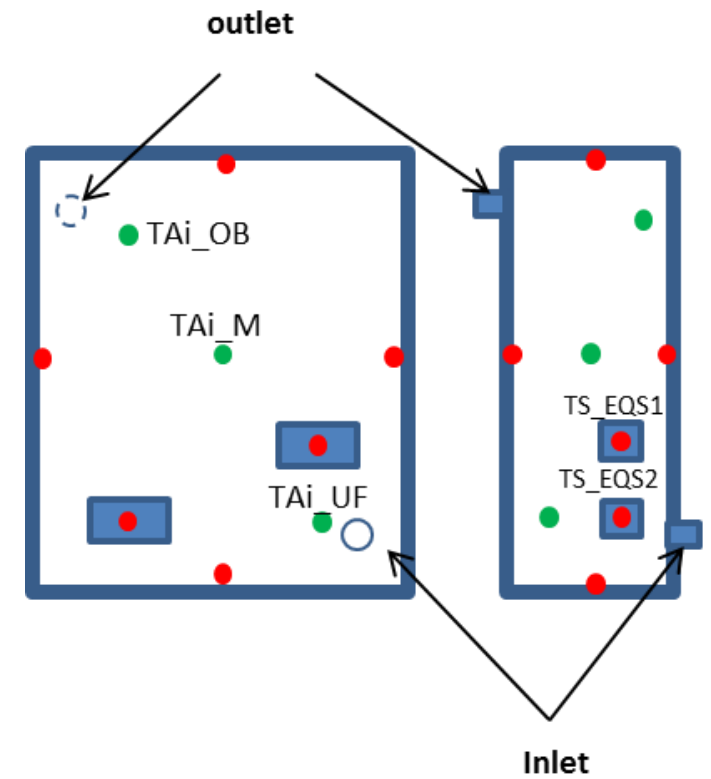

Figure 10: Sensor positions: green dots: location of air temperature sensors, red dots: location of surface temperature sensors

Figure 10 shows the distribution of measurement locations that were used for model validation in this study. Table 1 describes four different validation test cases varying supply airflow rate and long-wave emissivity of the EQS.

Table 1: Test Cases (TC1-4), Al: aluminium surface, B: black painted surface

\begin{tabular}{|c|c|c|c|c|}
\cline { 2 - 5 } \multicolumn{1}{c|}{} & TC1 & TC2 & TC3 & TC4 \\
\hline $\begin{array}{c}\text { Inlet mass flow } \\
\text { (kg/min) }\end{array}$ & 10 & 7.5 & 10 & 7.5 \\
\hline $\begin{array}{c}\text { Supply } \\
\text { Temperature (K) }\end{array}$ & 283.15 & 283.15 & 283.15 & 283.15 \\
\hline Pressure (hPa) & 940 & 940 & 940 & 940 \\
\hline Power EQS1 (W) & 500 & 500 & 500 & 500 \\
\hline Power EQS2 (W) & 500 & 500 & 500 & 500 \\
\hline Surface EQS1 & $\mathrm{Al}$ & $\mathrm{Al}$ & $\mathrm{B}$ & $\mathrm{B}$ \\
\hline Surface EQS2 & $\mathrm{Al}$ & $\mathrm{Al}$ & $\mathrm{Al}$ & $\mathrm{Al}$ \\
\hline
\end{tabular}




\subsection{Results}

To validate the coupled model, simulated results of four different test cases were compared with actual test results.

Figure 11 to Figure 13 show the comparison of simulated and measured air temperatures at the top left corner (TAi_OB), the center (TAi_M) and the bottom right corner of the box (TAi_UF) for TC1 and TC2. In steady-state conditions, the model predicts air temperature with less than $0.5 \mathrm{~K}$ deviations.

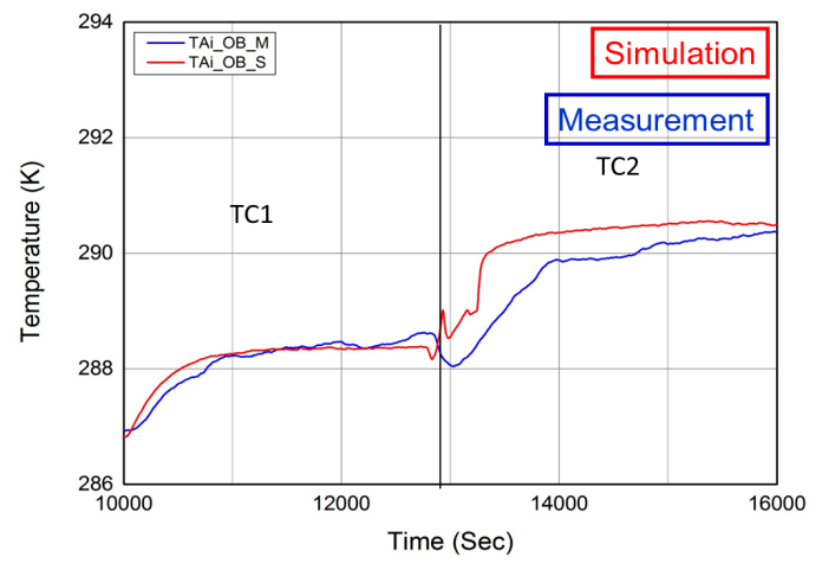

Figure 11: Air temperature at the top left corner of the box

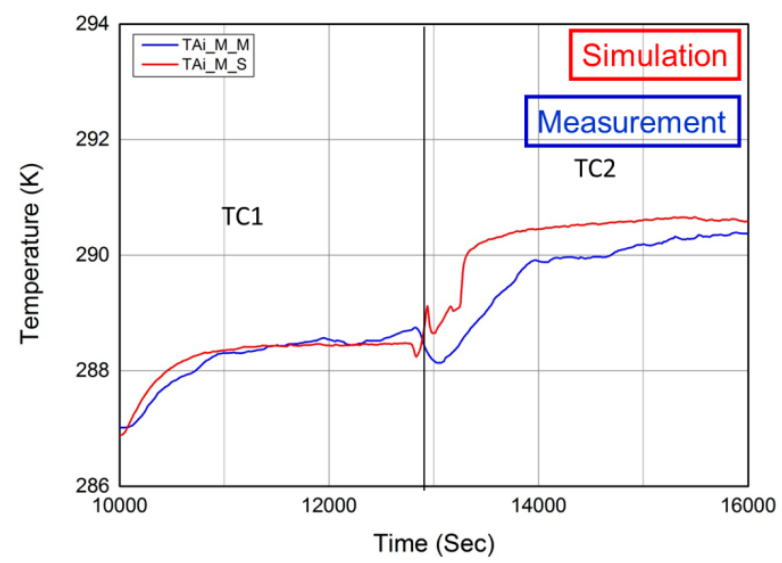

Figure 12: Air temperature at the center of the box

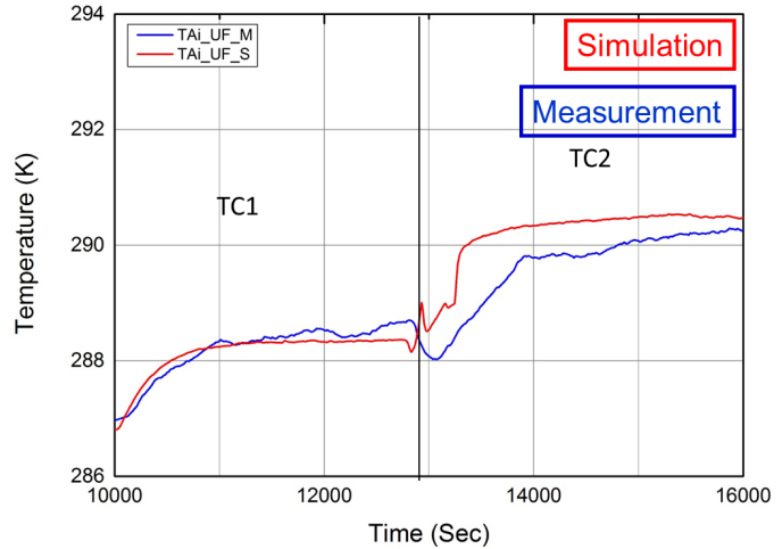

Figure 13: Air temperature at the bottom right corner of the box

Figure 14 and Figure 15 show the comparison of simulated and measured surface temperatures of EQS1 and EQS2. In the experimental setup, surface temperature sensors are placed on each face of the EQS. For model validation, these temperatures were averaged to obtain one representative surface temperature. At steady state, the deviation of measurement and simulation is less than $5 \mathrm{~K}$.

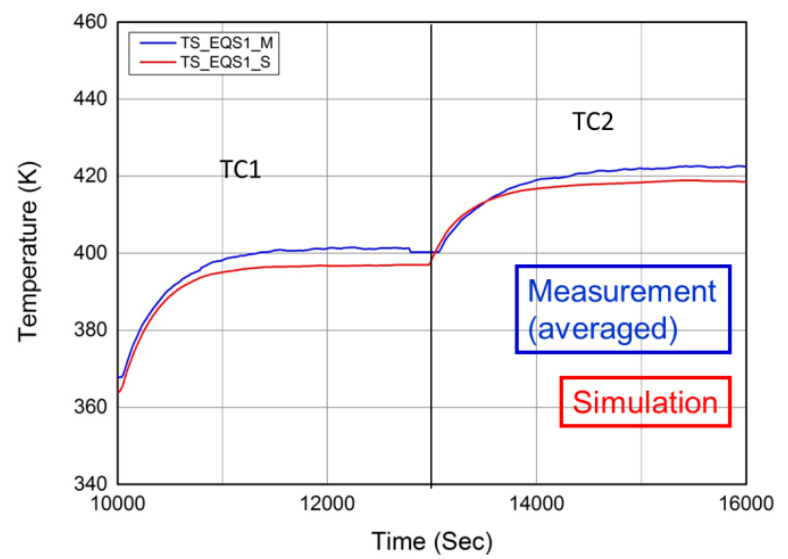

Figure 14: Surface temperature of EQS1 for TC1 and TC2

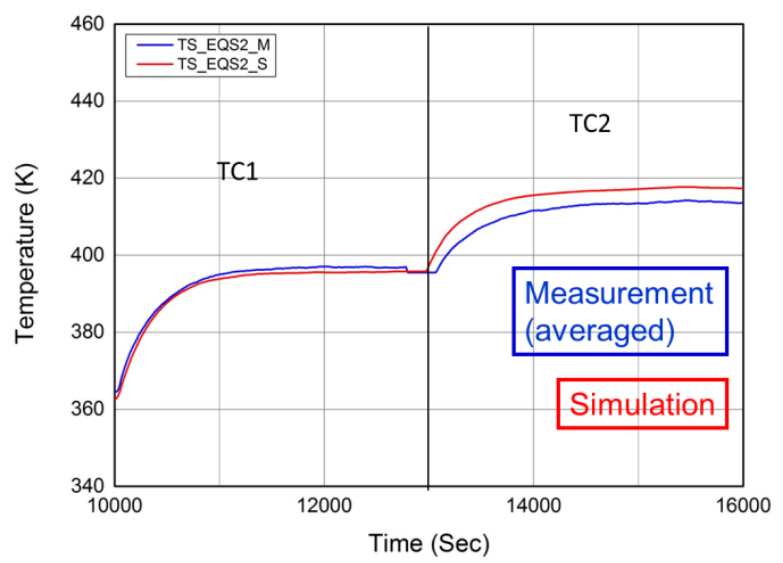

Figure 15: Surface temperature of EQS2 for TC1 and TC2 
To assess model validity for changed long-wave radiation properties, EQS1 was painted black for TC3 and TC4. Figure 16 and Figure 17 show the comparison of simulated and measured surface temperatures of EQS1 and EQS2. Due to higher radiative exchange, surface temperature of EQS1 is lower than that of EQS1 in TC1 and TC2. EQS2 receives more radiation from EQS1 resulting in a higher surface temperature compared to TC1 and TC2. The accuracy of model predictions is in the range of $5 \mathrm{~K}$ for this setup, too.

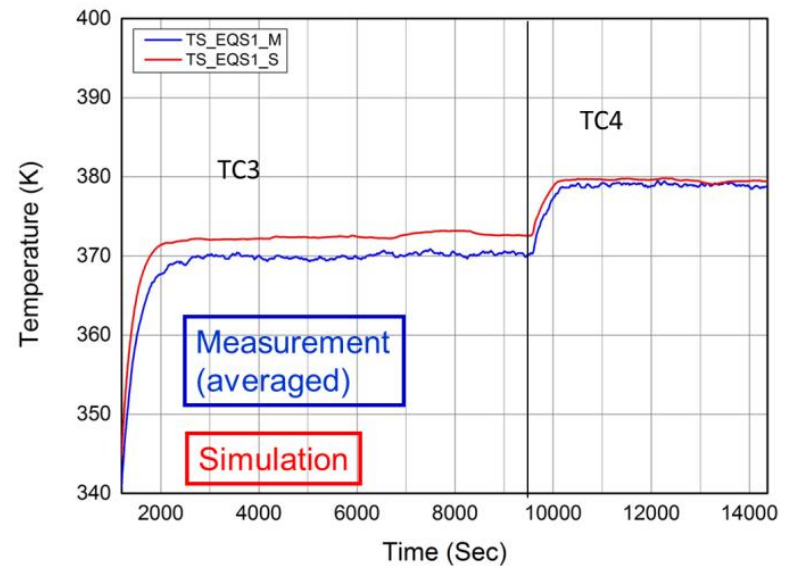

Figure 16: Surface temperature of EQS1 for TC3 and TC4

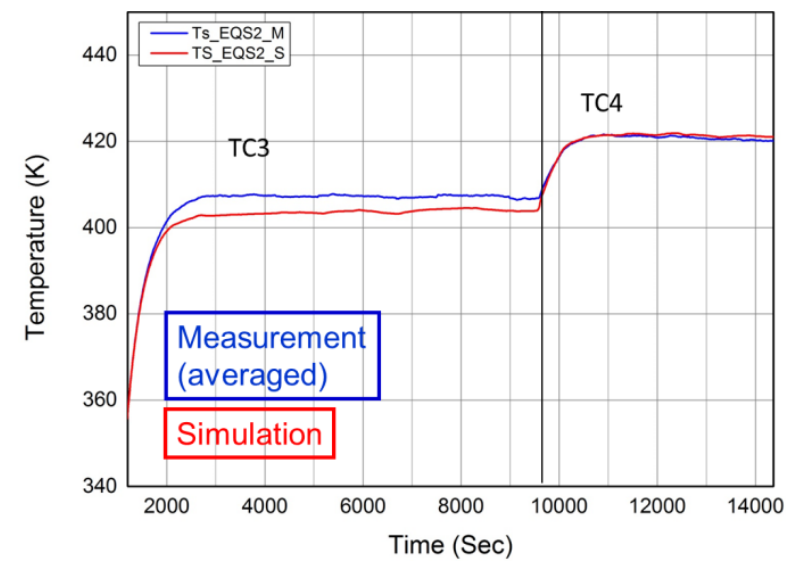

Figure 17: Surface temperature of EQS2 for TC3 and TC4

\subsection{Discussion}

Comparison with validation test data shows that results of air temperatures show slight variations during transients but are accurate in steady state. The model predicts the surface temperature of EQS well for both transient and steady state conditions and for different emissivity. The impact of a higher radiative loss of the black EQS might have been visible even in a simplified radiation approach. However, the relatively higher surface temperature of the other
EQS, still being low emissive, would not have been correctly predicted without the computed view factor matrix finding a considerable radiative heat exchange between both EQS.

\section{Application Examples}

This section shows two application examples that have been setup using the MThMGT.

\subsection{Radiant heating of a room}

In this example the MThMGT has been used to assess the impact of radiant heating. Figure 18 shows three sides of a room with a wall heater, a table and a sitting manikin. The table is located in the center of the room. It has been subdivided into ten parts, three on the right, three on the left and four on the lower side of the desk. The impact of using these parts as radiant heating sources by applying a heating foil on them is investigated. Zoning has been done such that each heat source lies in a separate zone.

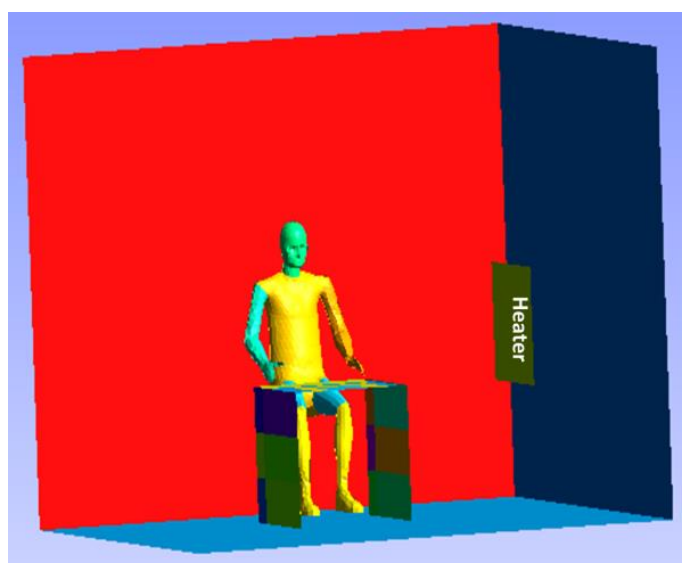

Figure 18: MThMGT application example of building room

Figure 19 shows the result of different parts of the manikin. The temperatures of legs and thighs are higher than other body parts because of the radiant heating from the heating foils on the inner sides of the table. The temperature of the left hand is higher than that of the right hand, as the left hand is closer and in line of the wall heater. The left part of the chest is cooler than the left arm even though being in line with the heater due to the obstruction caused by left arm. 


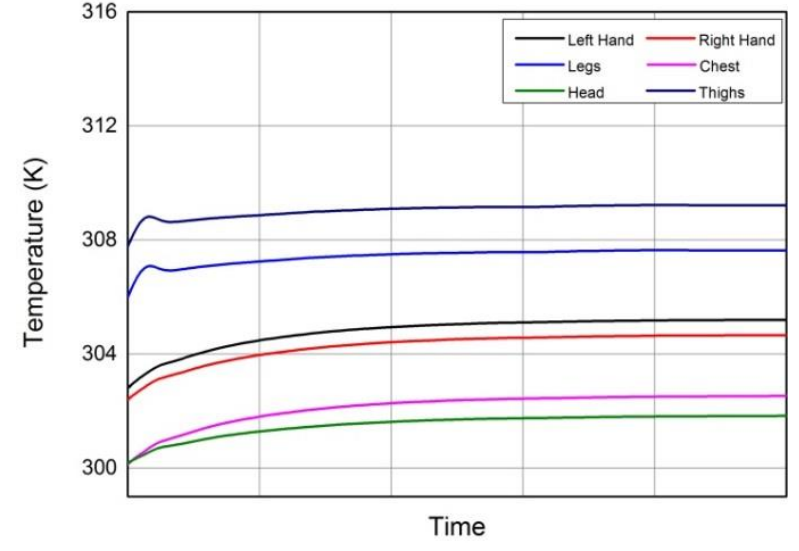

Figure 19: Temperatures of manikin body parts

\subsection{Cockpit Ventilation}

In order to determine the required ventilation performance of the aircraft air conditioning system, heat loads have to be balanced with the airflow requirements in the most challenging operating conditions. Major heat sources in the aircraft cabin are passengers, electrical devices, solar radiation, indirect heating from piping and additional driving factors such as hot and humid environmental conditions on ground or at low flight level. Lightings in the cabin and IFE are also major heat sources. The MThMGT allows investigating aircraft cooling strategies.

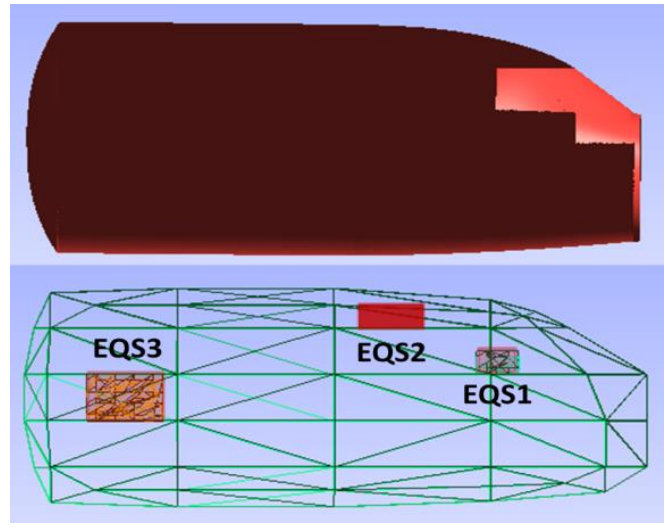

Figure 20: CAD and Zonal Grid of an aircraft cockpit

Figure 20 shows the CAD geometry of an aircraft cockpit (top). The cockpit is subdivided into $4 \times 3 \times 5$ zones assuring that each EQS is located in a separate zone (bottom). Heat productions of EQS1, EQS2 and EQS3 are set to 2.0, 1.0 and $1.5 \mathrm{~kW}$ respectively.

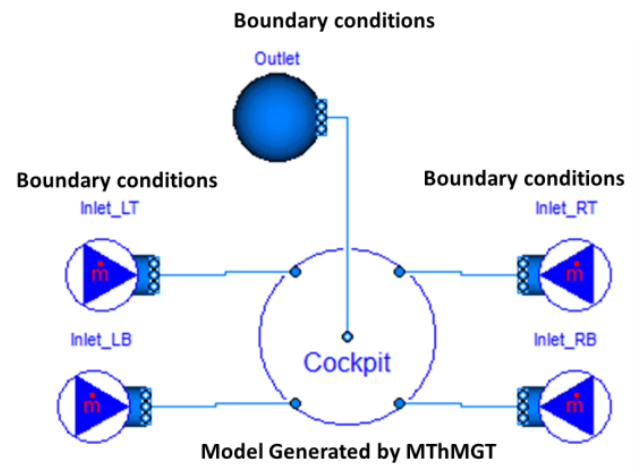

Figure 21: Auto-Generated Thermal Model

Figure 21 shows the generated thermal model connected to the airflow sources and the pressure sink. There are four airflow inlets in the cockpit: two at the top and two at the bottom. The outlet is located in the center of the rear side. The user needs to define boundary conditions such as inlet airflow rate, inlet air temperature, sink air pressure and exterior temperature and to select an appropriate wall model from a wall model package.

Simulation results are exported into the Paraview visualization format for post-processing [8]. Figure 22 shows simulated wall inner surface temperatures of the cockpit. The impact of radiation from equipment simulators is reflected in results. Facets $\mathrm{A}, \mathrm{B}$ and $\mathrm{D}$ are in close vicinity to EQS1 and EQS2. Facet $\mathrm{C}$ and $\mathrm{F}$ are influenced by EQS3. Facet $\mathrm{E}$ receives radiation from both EQS2 and EQS3.
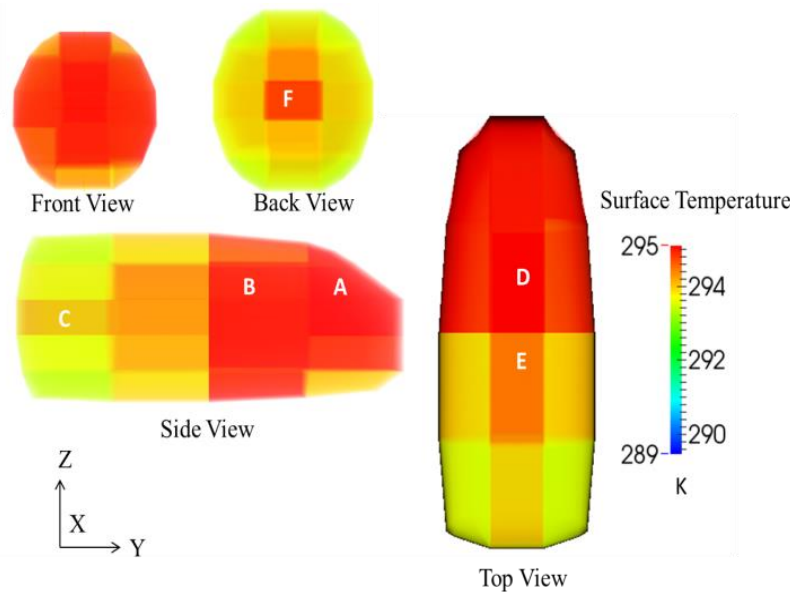

Figure 22: Wall inner surface temperatures

Figure 23 shows simulated air node temperatures in the cockpit. EQS1, EQS2 and EQS3 are located in zones $\mathrm{A}, \mathrm{B}$ and $\mathrm{C}$ respectively. In these zones, air temperature is predicted to be higher than in the other, empty zones. 


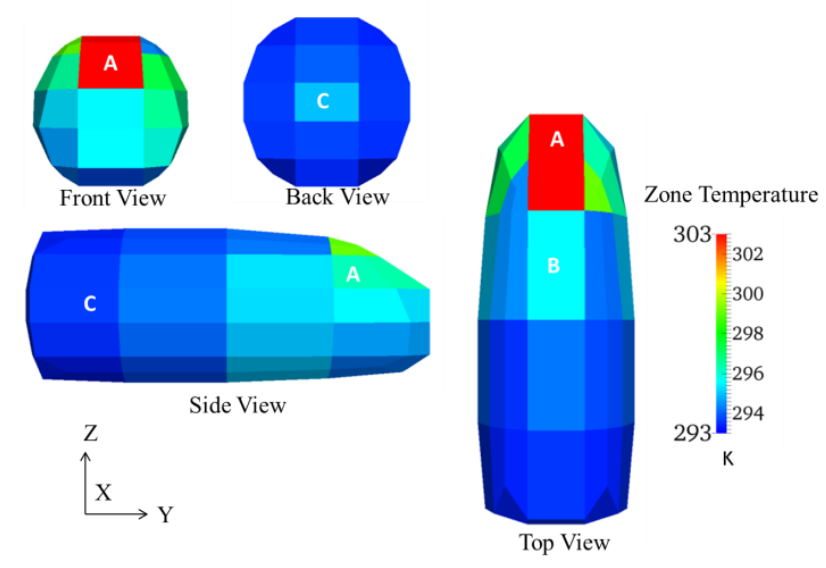

Figure 23: Air node temperatures

\section{Conclusion and Outlook}

A tool to model airflow and radiative heat transfer for complex geometries in 3D space has been outlined in the presented work. The Modelica Thermal Model Generation Tool closes the gap between the CAD model and the resulting Modelica thermal model. The exported model shows to correctly predict the interaction of radiative and convective heat transfer in an experimental validation case. The possibility to model the impact of radiative heating and to assess the impact of different airflow pattern on heating and cooling has been proved for both rectangular and irregular geometries.

The disadvantage of the generated code is that it is plain text with only scarce use of the graphical programming possibilities of Modelica. Further customization or changes in the model therefore require a high level of expertise of the user both in terms of the logics of the generated model and the code of contained models.

To keep the model accessible even for less experienced users, an export script allowing the visualization of simulation results in the open source post processing software ParaView has been written. From this visualization, the user can for example assess cooling and heating strategies or find local hot or cold spots.

For the future, we intend to include a model which can calculate convective heat transfer coefficients considering local zonal airflow pattern, surface properties and its orientation. This development provides a tool to predict thermal comfort in enclosed 3D spaces. This tool will allow for better thermal modelling when considering thermal management in buildings, automobiles or aircrafts.

\section{Acknowledgements}

This research is benefiting from the work that is being done in the European Community's Clean Sky JTI under grant agreement $n^{\circ}$ CSJU-GAM-ED-2008001.

\section{References}

[1] Norrefeldt, V., Andersson, D., Pathak, A. et al.: The Indoor Climate Library and its application to heat and moisture transfer in a vehicle cabin, $9^{\text {th }}$ Modelica Conference, Munich, Germany, 03.-05. september 2012

[2] Wetter, M.: Modelica library for building heating, ventilation and air-conditioning systems, $7^{\text {th }}$ International Modelica Conference, Como, Italy, 20.-22. september 2009

[3] Wetter, M.: Multizone airflow model in Modelica, $5^{\text {th }}$ Modelica Conference, Vienna, 4.-5.september 2006

[4] Bonvini, M., Leva, A.: Object-oriented subzonal room models for energy-related building simulation, $8^{\text {th }}$ Modelica Conference, 20.-22. march 2011, Dresden, Germany

[5] Wetter, M., Zuo, W., Nouidui, T.: Modeling of heat transfer in rooms in the Modelica "Buildings" Library, Building Simulation, Sydney, Australia, 14.-16. november 2011

[6] Norrefeldt, V., Grün, G.: VEPZO - Velocity Propagating Zonal Model for the prediction of airflow pattern and temperature distribution in enclosed spaces, $9^{\text {th }}$ Modelica Conference, Munich, Germany, 03.-05. september 2012

[7] Pathak, A., Norrefeldt, V., Grün, G.: Modelling of radiative heat transfer in Modelica with a mobile solar radiation model and a view factor model, $9^{\text {th }}$ Modelica Conference, September 3-5, Munich, Germany

[8] Paraview: Paraview, www.paraview.org, page consulted 05.12.2013, 